\title{
Muscle damage responses and adaptations to eccentric-overload resistance exercise in men and women
}

\author{
Rodrigo Fernandez-Gonzalo • Tommy R. Lundberg • \\ Lucia Alvarez-Alvarez • José A. de Paz
}

Received: 20 September 2013 / Accepted: 25 January 2014 / Published online: 12 February 2014

(C) Springer-Verlag Berlin Heidelberg 2014

\begin{abstract}
Purpose This study assessed markers of muscle damage and training adaptations to eccentric-overload flywheel resistance exercise (RE) in men and women.

Methods Dynamic strength (1 RM), jump performance, maximal power at different percentages of $1 \mathrm{RM}$, and muscle mass in three different portions of the thigh were assessed in 16 men and 16 women before and after 6 weeks (15 sessions) of flywheel supine squat RE training. Plasma creatine kinase $(\mathrm{CK})$ and lactate dehydrogenase (LDH) concentrations were measured before, 24, 48 and $72 \mathrm{~h}$ after the first and the last training session.

Results After training, increases in 1 RM were somewhat greater (interaction $P<0.001)$ in men $(25 \%)$ than in women $(20 \%)$. Squat and drop jump height and power performance at 50, 60, 70 and $80 \%$ of $1 \mathrm{RM}$ increased after training in both sexes $(P<0.05)$. Power improvement at $80 \%$ of $1 \mathrm{RM}$ was greater (interaction $P<0.02$ ) in men than women. Muscle mass increased $\sim 5 \%$ in both groups $(P<0.05)$. CK increased in men after the first training session $(P<0.001)$, whereas the response in women was unaltered. In both sexes, LDH concentration was greater
\end{abstract}

Communicated by Peter Krustrup.

R. Fernandez-Gonzalo $(\square)$

Department of Physiology and Pharmacology, Karolinska

Institutet, 17177 Stockholm, Sweden

e-mail: rodrigo.fernandez.gonzalo@ki.se

R. Fernandez-Gonzalo $\cdot$ L. Alvarez-Alvarez · J. A. de Paz Institute of Biomedicine (IBIOMED), University of León, León, Spain

T. R. Lundberg

Department of Health Sciences, Mid Sweden University,

Östersund, Sweden after the first training session compared with basal values $(P<0.05)$. After the last session, $\mathrm{CK}$ and $\mathrm{LDH}$ remained at baseline in both groups.

Conclusions These results suggest that although improvements in maximal strength and power at high loads may be slightly greater for men, eccentric-overload RE training induces comparable and favorable gains in strength, power, and muscle mass in both men and women. Equally important, it appears muscle damage does not interfere with the adaptations triggered by this training paradigm.

Keywords $1 \mathrm{RM} \cdot$ Power $\cdot$ Muscle hypertrophy $\cdot \mathrm{CK}$. $\mathrm{LDH}$

$\begin{array}{ll}\text { Abbreviations } \\ \text { ANOVA } & \text { Analysis of variance } \\ \text { CK } & \text { Creatine kinase } \\ \text { CMJ } & \text { Countermovement jump } \\ \text { CON } & \text { Concentric } \\ \text { DJ } & \text { Drop jump } \\ \text { DXA } & \text { Dual energy X-ray absorptiometry } \\ \text { ECC } & \text { Eccentric } \\ \text { LDH } & \text { Lactate dehydrogenase } \\ \text { MRI } & \text { Magnetic resonance imaging } \\ \text { RE } & \text { Resistance exercise } \\ \text { RM } & \text { Repetition maximum } \\ \text { SEM } & \text { Standard error of the mean } \\ \text { SJ } & \text { Squat jump }\end{array}$

\section{Introduction}

Resistance exercise (RE) training increases muscle mass and strength in both men and women, yet there is no consensus whether the magnitude of these adaptations differs 
across sexes. Thus, some studies have shown differences in RE-induced gains in muscle size, force and power across sexes (Colliander and Tesch 1991; Hubal et al. 2005; Shephard 2000), whereas others have not (Abe et al. 2000; Staron et al. 1994). More specifically, increases in muscle mass (Hubal et al. 2005; Melnyk et al. 2009; Shephard 2000) and peak power in the slow-speed, high-force region (Colliander and Tesch 1991) may be greater for men than women. Although the reason for these potential discrepancies across sexes is largely unknown, differences in the endocrine, neuromuscular or cellular response to highintensity exercise has been put forth as tentative explanations (Billaut and Smith 2009; Esbjornsson et al. 2012; Kraemer et al. 1998).

Skeletal muscle inherently has greater capacity to produce force during eccentric (ECC) than concentric (CON) muscle actions. Even though ECC compared with $\mathrm{CON}$ actions impose a lower metabolic demand on the muscle (Dudley et al. 1991a), they have been shown imperative for optimal gains in strength (Colliander and Tesch 1990, 1992) and muscle mass (Hather et al. 1991; Norrbrand et al. 2008) in response to RE training. Thus, to optimize the effects of resistance training, eccentric muscle actions should be included (Dudley et al. 1991b).

Traditional CON-ECC weight training employs constant external load, inevitably resulting in submaximal ECC actions. In contrast, isoinertial exercise could offer maximal voluntary resistance during both CON and ECC actions. To meet this requirement, an exercise system $\left(\mathrm{YoYo}^{\circledR}\right.$ Technology, Stockholm, Sweden) which allowed for coupled CON and ECC muscle actions through inertial resistance from a spinning flywheel, was developed. By means of specific instructions to the trainee, this exercise paradigm allows for brief episodes of ECC overload (Berg and Tesch 1994; Tesch et al. 2004). Apart from the obvious applicability in combating the deleterious effects on muscle tissue during spaceflights (Alkner and Tesch 2004a, b; Trappe et al. 2007), this gravity-independent RE system has also been shown effective during injury prevention and rehabilitation (Askling et al. 2003; Romero-Rodriguez et al. 2011). Thus, due to its unique loading features, flywheel RE, employing ECC overload, induces early and robust increases in strength and muscle mass that are greater than those realized with conventional RE training (Norrbrand et al. 2008, 2010, 2011). To date, however, most studies incorporating ECC-overload RE training have been conducted in men. Potential difference in muscle adaptive responsiveness across sexes, therefore, remains to be elucidated.

While proven effective in inducing robust muscle adaptations, ECC muscle actions may transiently decrease maximal strength (Golden and Dudley 1992) and generate muscle damage (Allen 2001; Garcia-Lopez et al. 2007; Milias et al. 2005). More specifically, unaccustomed ECC exercise produces sarcolemma disruption and inflammatory responses, which is manifested by increases in established muscle damage markers such as creatine kinase (CK) and lactate dehydrogenase (LDH) in the circulation (Barroso et al. 2010; Chen et al. 2009). If long-lasting, muscle damage could serve as an adverse feature of training leading to compromised training adaptations and increased proteolytic activity (Cannon et al. 1991; Krentz and Farthing 2010; Thompson and Scordilis 1994; Stupka et al. 2001). While short- and long-term traditional RE training typically blunts the exercise-induced muscle damage response (FernandezGonzalo et al. 2012; Garcia-Lopez et al. 2007; Nosaka and Newton 2002; Ploutz-Snyder et al. 2001; Schroeder et al. 2004), it remains to be shown whether (1) flywheel ECCoverload RE triggers muscle damage responses, (2) if these responses are attenuated with training, and (3) if these adaptations are comparable across sexes.

The aim of this study was to assess and compare adaptations in muscle strength, power, and mass after a short-term (6 weeks) ECC-overload RE training program in men and women. In addition, muscle damage responses were examined after the first and last training session. We hypothesized that improvements in strength, power and muscle mass would be similar across sexes, and that training would blunt acute increases in markers of muscle damage in both men and women.

\section{Methods}

\section{General design}

Thirty-two healthy subjects (16 men and 16 women) completed 6 weeks (15 sessions) of bilateral flywheel supine squat RE. Each session consisted of four sets of seven maximal repetitions performed 2-3 times week ${ }^{-1}$. Maximal dynamic strength (1 RM), vertical jump performance, power at different loads of $1 \mathrm{RM}$ and thigh muscle mass were assessed approximately 1 week before and after the training period (Fig. 1). In addition, CK and LDH plasma concentrations were measured from blood samples obtained before, 24,48 and $72 \mathrm{~h}$ after the first and the last training session.

\section{Subjects}

Thirty-two sports science undergraduate students (16 men; $23 \pm 1$ years, $75 \pm 2 \mathrm{~kg}$ and $178 \pm 2 \mathrm{~cm}$, and 16 women; $24 \pm 1$ years, $59 \pm 1 \mathrm{~kg}$ and $164 \pm 2 \mathrm{~cm}$; mean \pm standard error of the mean; SEM) volunteered for the study. Subjects were moderately active and healthy individuals, engaged in 4-6 h of recreational exercise per week. They had no history of lower limb strength training for the past 


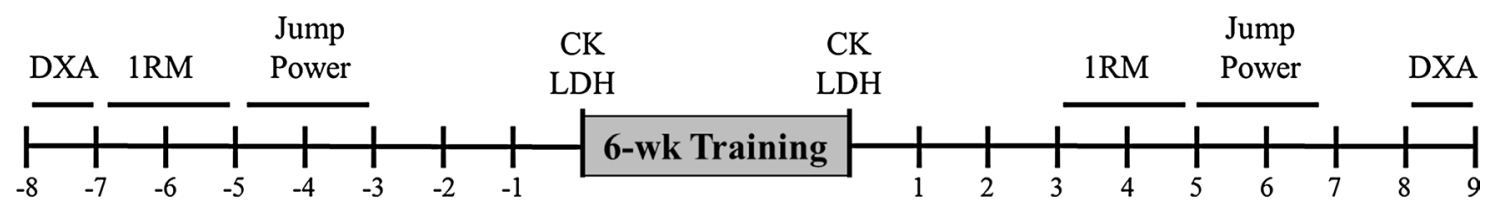

Days

Fig. 1 Schematic overview of the study protocol. $D X A$ dual energy X-ray absorptiometry, $1 R M$ one-repetition maximum, $C K$ creatine kinase, $L D H$ lactate dehydrogenase. $\mathrm{CK}$ and $\mathrm{LDH}$ were assessed before and 24,48 and $72 \mathrm{~h}$ after the first and the last training sessions

6 months and no previous muscle joint or bone injury. The female subjects did not use hormonal contraceptives. Subjects were informed of the purposes and risks involved in the study before giving their informed written consent to participate. The study protocols were approved by the Ethics Committee of the University of León. All subjects complied with two familiarization sessions, the prescribed training program, and the pre- and post-tests.

Maximal dynamic strength, jump performance and power tests

The $1 \mathrm{RM}$ test was conducted on a $45^{\circ}$ inclinated leg press device (Gerva-Sport, Madrid, Spain). Subjects performed one repetition from $90^{\circ}$ to full extension $\left(180^{\circ}\right)$ with a load of $\sim 3 \mathrm{RM}$. The load was increased with $10 \mathrm{~kg}$ if the subject succeeded or decreased $5 \mathrm{~kg}$ if failed. Testing ended when subjects failed to overcome a given load in two successive trials. 1 RM was achieved between three and six attempts, and trials were interspersed by 3 -min recovery. The $1 \mathrm{RM}$ test was performed twice; 5-7 days before and 3-5 days after the training period (Fig. 1). A warm-up of 5-min cycling and one set of $\sim 8 \mathrm{RM}$ preceded the 1 RM test.

Vertical jump tests were carried out 3-4 days before the first training session and 6-7 days after the last training session (Fig. 1). Jump height was measured for three types of jumps performed on a contact platform (Globus Ergotester $^{\circledR}$, Globus, Codogne, Italy): (1) squat jump (SJ), (2) countermovement jump (CMJ), and (3) drop jump (DJ). The SJ was initiated from a $90^{\circ}$ knee flexion with hands at the hips. The subject jumped as high as possible, without any downward countermovement prior to the upward movement, and landed with straight legs on the platform. For the CMJ, the subject started in a standing straight position and was instructed to jump as high as possible with hands at the hips. The DJ consisted of dropping oneself from a box of $40 \mathrm{~cm}$, jumping as high as possible straight after landing. Jump height was recorded to the nearest $0.1 \mathrm{~cm}$. Three trials, interspersed with 1-min recovery, were allowed and the best result was included in the data analysis.

Fifteen min after the jump tests, power was assessed on the same inclinated leg press employed for the $1 \mathrm{RM}$ test.
Subjects completed five sets of three repetitions from $90^{\circ}$ knee flexion to full extension $\left(180^{\circ}\right)$ with 3-min recovery between sets. To avoid any use of the stretch-shortening cycle, each repetition started from a complete static position. Each set represented 40, 50, 60, 70, and $80 \%$ of $1 \mathrm{RM}$, and the order of the sets was individually randomized before training and replicated at post-tests. Subjects were requested to perform the CON phase of each repetition as fast as possible. Average and peak power for each repetition were sampled at $100 \mathrm{~Hz}$ using an encoder (SmartCoach ${ }^{\mathrm{TM}}$, SmartCoach Europe AB, Stockholm, Sweden) and associated software (SmartCoach ${ }^{\circledR}$ v.3.1.8.0). The best repetition performed at each load was used for data analysis.

\section{Dual energy X-ray absorptiometry analysis}

Dual energy X-ray absorptiometry (DXA) was performed twice on the same time of the day, 1 week before and after the training program (Fig. 1) using a Lunar Prodigy ${ }^{\circledR}$ whole-body scanner (GE Medical Systems, Madison, WI). Once all data were stored, a manual analysis was performed to estimate thigh muscle mass (Encore ${ }^{\circledR} 2009$ software, Lunar Corp., Madison, WI). Briefly, two horizontal line marks were generated using the lower margin of the ischial tuberosities and the lower margin of the femoral condyles as reference points. Subsequently, a perpendicular line was drawn from the distal to the proximal mark. The length of this line was calculated and a 20-mm-thick slice was placed in the mid-thigh of one leg (Hansen et al. 2007). In addition, two other 20-mm slices were placed $5 \mathrm{~cm}$ above (upper thigh) and $5 \mathrm{~cm}$ below (lower thigh) the first mark (Fig. 2). Finally, the software was configured to calculate lean tissue (i.e., muscle mass; g) in the slices created. The legs chosen for pre/post analyses were randomized in a counterbalanced manner, but conducted on the same leg for each subject. The error attributable to manual positioning of the mid-thigh region, assessed from repeated analysis of the scans of ten subjects, was $1.5 \%$. Although magnetic resonance imaging (MRI) is the reference method to assess muscle volume, results obtained through DXA analysis correlate with MRI and (Andreoli et al. 2009; Hansen et al. 2007) represents a cheaper and less time-consuming 


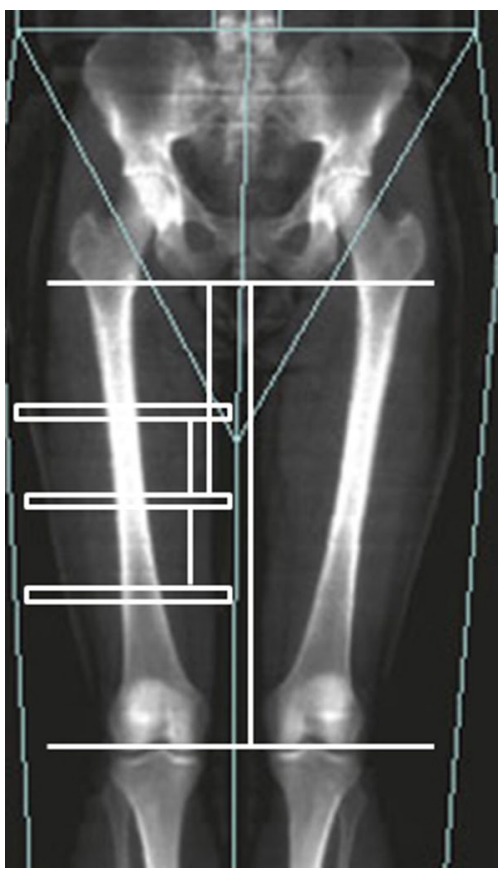

Fig. 2 Scheme of the three different "slices" used to estimate thigh muscle mass by DXA (left thigh is shown)

approach to estimate muscle mass. However, a limitation of this technique is the impossibility to differentiate between individual muscles.

\section{Plasma CK and LDH concentration}

Venous blood samples $(5 \mathrm{~mL})$ were drawn from the brachiocephalic vein before and 24, 48 and $72 \mathrm{~h}$ after the first and the last training session, using the Vacutainer ${ }^{\mathrm{TM}}$ system (BD, Franklin Lakes, NJ) with heparinized tubes. Samples were centrifuged for $10 \mathrm{~min}$ to obtain plasma. CK and LDH concentrations were measured spectrophotometrically (Cobas Integra ${ }^{\circledR} 400$, Roche, Basel, Switzerland) using specific liquid reagents (Cobas Integra ${ }^{\circledR}$, Roche, Basel, Switzerland). The reference range with this method is $\leq 170$ and $\leq 142 \mathrm{IU} \mathrm{L}^{-1}$ for CK in men and women, respectively, and 135-225 and 135-214 IU L ${ }^{-1}$ for LDH.

\section{Training program}

Subjects performed supine squat training using a MultiGym flywheel device (YoYo ${ }^{\mathrm{TM}}$ Technology Inc, Stockholm, Sweden) (Norrbrand et al. 2011), equipped with two $2.7-\mathrm{kg}$ flywheels with a moment inertia of $0.07 \mathrm{~kg} \mathrm{~m}^{-2}$ each. Subjects trained 2 (week 1, 3, 5) or 3 (week 2, 4, 6) times week ${ }^{-1}$ with at least $48 \mathrm{~h}$ of rest between sessions. Following a standardized cycling warm-up, subjects performed four sets of seven maximal bilateral coupled CON and ECC muscle actions in the supine squat device. An extra repetition was performed at the beginning of each set to initiate flywheel movement. The main seven repetitions were performed with maximal effort, accelerating the wheel in the CON action and upon completion, decelerating the wheel by means of an ECC action. Subjects were requested to push with maximal effort through the entire CON action, which ranged from $70^{\circ}$ knee flexion to near full extension. At the end of the CON action, the flywheel strap winds back due to inertial forces, which initiates the reversed ECC action. During the first third of the ECC action, subjects were instructed to resist gently, and thereafter to apply maximal breaking force to stop the movement at about $70^{\circ}$ knee flexion. By means of this approach, ECC overload is produced (Norrbrand et al. 2010; RomeroRodriguez et al. 2011; Tesch et al. 2004). Power was measured during each repetition (CON and ECC actions; SmartCoach ${ }^{\mathrm{TM}}$ ) and real-time feedback was provided on a computer monitor. Research personnel gave strong verbal encouragement during all repetitions performed. All subjects were familiarized ( 2 sessions) with the flywheel supine squat exercise prior to the first training session.

\section{Statistical analysis}

Statistical analyses were performed using SPSS v.20.0 (SPSS Inc. Chicago, IL). Results are expressed as mean \pm SEM. Data distribution was examined for normality using the Shapiro-Wilk test. One-repetition maximum, squat jump, countermovement jump, drop jump and power at the different loads measured were examined using a twoway ANOVA (sex $\times$ training). $\mathrm{CK}$ and $\mathrm{LDH}$ concentrations after the first and the last training sessions were analyzed by a three-way ANOVA (sex $\times$ training $\times$ time). When a significant interaction was found, post hoc comparisons with Bonferroni adjustment were employed. The significance level was set to $P<0.05$.

\section{Results}

There was a significant main effect of sex for all variables analyzed ( $F$ range 27.3-172.8), except for LDH concentration, which showed a tendency towards an effect of sex $(P=0.068)$. Thus, men showed greater absolute values in $1 \mathrm{RM}$, jump performance, power, muscle mass and CK concentration before and after training (all $P<0.05$ ) compared with women. Because sex differences in absolute values were so evident, they are not indicated in tables or figures unless specifically stated. While CON and ECC power during training (Fig. 3) were markedly higher in men $(P<0.001)$, the relative increase did not differ between men and women $(P>0.05)$. Across sessions, $\mathrm{CON}$ power 


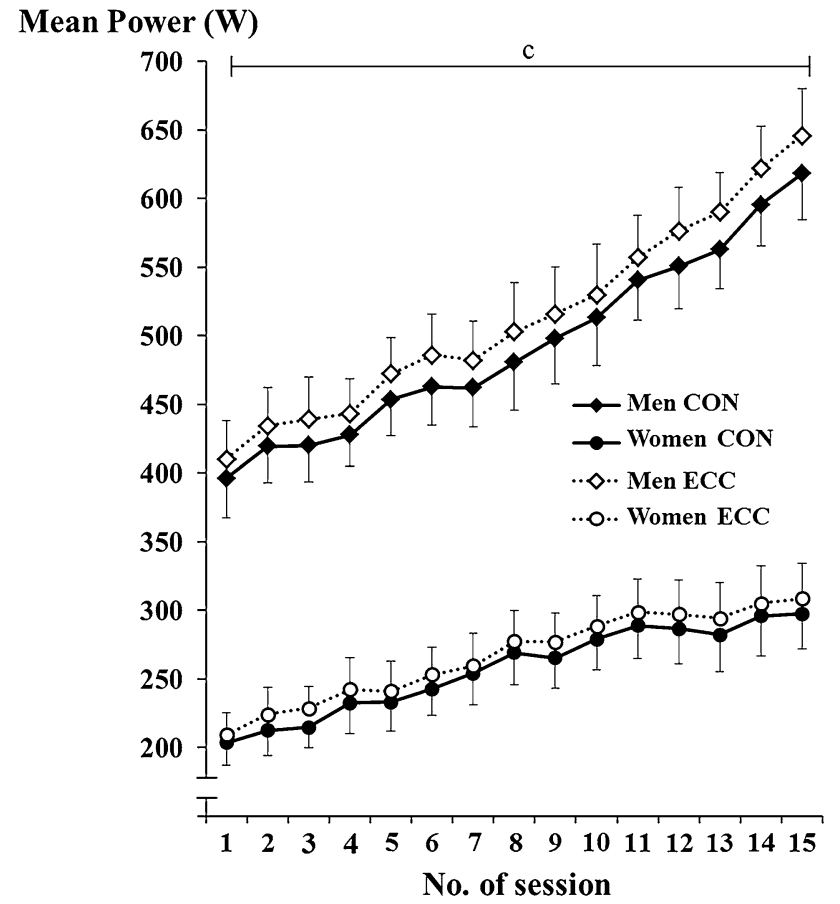

Fig. 3 Mean concentric (CON) and eccentric (ECC) power during each training session in men and women. Significant effects $(P<0.05): c$ main effect of time

increased 56 and $46 \%$ in men and women, respectively. The corresponding increases in ECC power were $57 \%$ in men and $47 \%$ in women. Peak power during the first training session was $718 \pm 175$ and $730 \pm 170 \mathrm{~W}$ for CON and ECC actions in men, and $366 \pm 121$ and $374 \pm 116 \mathrm{~W}$ for CON and ECC actions in women. Across sessions, increases in peak power were comparable between sexes (men 49 and $54 \%$ for CON and ECC actions, respectively; women 47 and $48 \%$ for CON and ECC actions, respectively). The ECC overload produced ( $\%$ above $\mathrm{CON}$ power) was 4.2 and $4.4 \%$ for men and women, respectively, with no difference between sessions or sexes. In other studies from our laboratory, this overload expressed in power, corresponds to a $15-30 \%$ ECC overload in force values $(N)$.
Maximal dynamic strength, jump performance and power tests

There was a sex $\times$ training interaction for $1 \mathrm{RM}(F=13.9$; $P<0.001)$ due to a greater $1 \mathrm{RM}$ increase in men than women (Table 1). Thus, 1 RM increased $25 \%$ in men and $20 \%$ in women after flywheel training $(P<0.001$ for both sexes).

No interaction was found in any of the jump protocols. However, there was a main effect of training in SJ $(F=4.4, P=0.046)$ and DJ $(F=5.0, P=0.034)$ because both men $(\sim 4 \%)$ and women $(\sim 8 \%)$ increased jump height after training.

There was no sex $\times$ training interaction for power performance (Fig. 4) at any load, with the exception that men showed greater improvements than women at $80 \%$ of $1 \mathrm{RM}(F=6.7, P=0.015)$. There was a main effect of training for all loads apart from $40 \%$ of $1 \mathrm{RM}$. Before training, maximal power was reached at the load corresponding to $70 \%$ of $1 \mathrm{RM}$ by both men and women whereas after the training period, maximal power was reached at $80 \%$ of $1 \mathrm{RM}$ by both groups.

\section{Muscle mass}

There was no interaction in any of the muscle mass estimations. However, there was a main effect of training in the three sections measured and in the sum of them (Table 2). Muscle mass increased $(P<0.01)$ after training in men (3.8\% for upper thigh, $5.7 \%$ for middle thigh, $4.7 \%$ for lower thigh, and $4.6 \%$ for summation). Likewise, women showed increased muscle mass $(P<0.001)$ in the upper $(5.0 \%)$, middle $(6.0 \%)$, and lower thigh (5.2\%), as well as in the sum of these sections $(5.4 \%)$.

\section{$\mathrm{CK}$ and $\mathrm{LDH}$ concentration}

There was a sex $\times$ time interaction for CK $(F=5.3$, $P<0.005)$. While CK concentration remained unchanged across time points in women, it increased in men $48 \mathrm{~h}$ after

Table 1 One repetition maximum (1 RM) and jump height in men and women pre- and post-training

\begin{tabular}{|c|c|c|c|c|c|c|}
\hline & \multicolumn{3}{|l|}{ Men } & \multicolumn{3}{|l|}{ Women } \\
\hline & Pre & Post & $\Delta \%$ & Pre & Post & $\Delta \%$ \\
\hline $1 \mathrm{RM}(\mathrm{kg})^{\mathrm{a}, \mathrm{b}}$ & $279.7 \pm 9.2$ & $350.2 \pm 10.1^{\#}$ & $25^{*}$ & $191.9 \pm 10.6$ & $229.4 \pm 17.9^{\#}$ & 20 \\
\hline $\mathrm{SJ}(\mathrm{cm})^{\mathrm{b}}$ & $34.4 \pm 1.1$ & $35.9 \pm 1.6$ & 4 & $25.1 \pm 1.4$ & $27.0 \pm 1.2$ & 8 \\
\hline $\mathrm{CMJ}(\mathrm{cm})$ & $40.5 \pm 1.2$ & $41.8 \pm 2.6$ & 3 & $28.9 \pm 0.9$ & $30.7 \pm 1.1$ & 6 \\
\hline $\mathrm{DJ}(\mathrm{cm})^{\mathrm{b}}$ & $38.6 \pm 1.1$ & $39.7 \pm 1.3$ & 3 & $26.5 \pm 1.0$ & $29.3 \pm 1.1$ & 11 \\
\hline
\end{tabular}

Values are mean \pm SEM. Significant effects $(P<0.05)$ : ${ }^{\mathrm{a}}$ sex $\times$ training interaction, ${ }^{\mathrm{b}}$ main effect of training. Significant post hoc differences $(P<0.05)$ : * vs women; ${ }^{\#}$ vs Pre within a sex

$S J$ squat jump, $C M J$ counter movement jump, $D J$ drop jump 


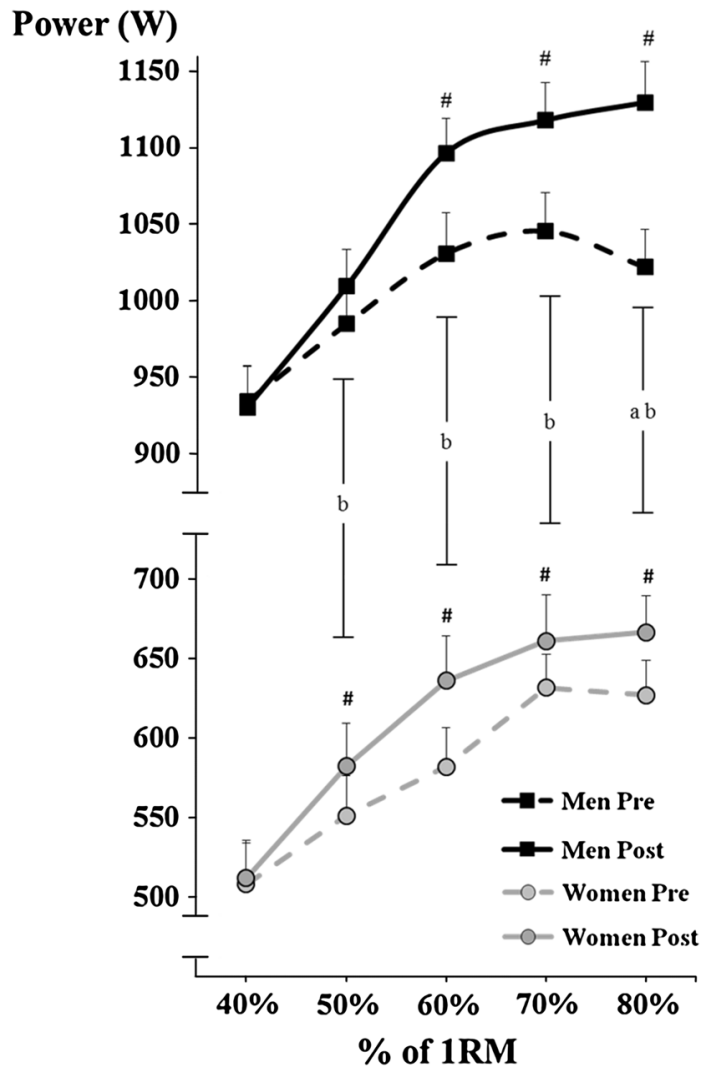

Fig. 4 Maximal leg press power at different percentages of $1 \mathrm{RM}$ before and after training. Significant effects $(P<0.05): a$ interaction (sex $\times$ training), $b$ main effect of training. Significant post hoc differences $(P<0.05)$ : ${ }^{\#}$ vs Pre within a sex

the first training session (Fig. 5). Additionally, there was a training $\times$ time interaction $(F=4.9, P<0.005)$ for $\mathrm{CK}$ because of the increased concentration in men 24,48 , and $72 \mathrm{~h}$ after the first training session $(P<0.001)$. In addition, values after the first training session were higher than after the last (main effect: $F=6.8, P<0.02$ ). Thus, men showed lower CK values 24,48 , and $72 \mathrm{~h}$ after the last training session compared with the first $(P<0.03)$.
LDH showed a training $\times$ time interaction $(F=5.8$, $P<0.003)$ because of the increased concentration in both men and women $48 \mathrm{~h}$ after the first training session ( $P<0.001$ for men and $P<0.05$ for women), but not after the last (Fig. 5). Furthermore, increases in LDH concentration were attenuated by training (main effect: $F=34.5$, $P<0.001)$ in both men and women. Both groups showed lower LDH concentration 24, 48 and $72 \mathrm{~h}$ after the last training session compared with the first $(P<0.02$ for men, $P<0.05$ for women).

\section{Discussion}

This is the first study comprehensively examining sexbased differences with regards to muscle damage responses and training adaptations induced by short-term ECC-overload RE training. The 6-week training paradigm produced comparable increases in strength, power and muscle mass across sexes, even though men showed slightly greater improvements in $1 \mathrm{RM}$ and power at $80 \%$ of $1 \mathrm{RM}$, than women. CK concentration after the first training session was greater in men than in women, yet blood markers of muscle damage were attenuated in both sexes after the last training session. Collectively, the results suggest that key adaptations prompted by short-term ECC-overload RE training are comparable between sexes and importantly, since no adverse effects from muscle damage were evident, it appears both men and women tolerate ECC-overload training.

ECC exercise is associated with muscle damage (Allen 2001; Garcia-Lopez et al. 2007; Milias et al. 2005). In the current study, the first training session induced muscle damage responses in both sexes. However, men showed greater increases in $\mathrm{CK}$, confirming previous investigations (Stupka et al. 2000). It has been suggested that this response is due to a greater muscle mass involved in the exercise (Mastaloudis et al. 2006). However, even though thigh muscle mass was 1.6-fold greater in men than in

Table 2 Estimated muscle mass ( $\mathrm{g}$ ) in the different portions (upper, middle, lower) of the thigh and the sum of them in men and women preand post-training

\begin{tabular}{llllr}
\hline & Upper & & Lower $^{\mathrm{a}}$ & Total $^{\mathrm{a}}$ \\
\hline Men & & & & \\
Pre & $427.7 \pm 10.2$ & $348.2 \pm 13.3$ & $259.3 \pm 14.0$ & $985.4 \pm 63.6$ \\
Post & $444.1 \pm 11.0^{\#}$ & $368.0 \pm 12.5^{\#}$ & $271.6 \pm 14.2^{\#}$ & $1,031.1 \pm 64.4^{\#}$ \\
Women & & & \\
Pre & $289.3 \pm 11.6$ & $222.5 \pm 7.8$ & $152.9 \pm 5.8$ & $596.4 \pm 47.6$ \\
Post & $303.8 \pm 17.6^{\#}$ & $235.7 \pm 11.3^{\#}$ & $160.9 \pm 8.2^{\#}$ & $628.5 \pm 50.2^{\#}$ \\
\hline
\end{tabular}

Values are expressed as mean \pm SEM. Results under total represent the sum of the three thigh parts measured. Significant effects $(P<0.05)$ :

${ }^{a}$ main effect of training. Significant post hoc differences $(P<0.05)$ : ${ }^{\#} \mathrm{vs}$ Pre within a sex 


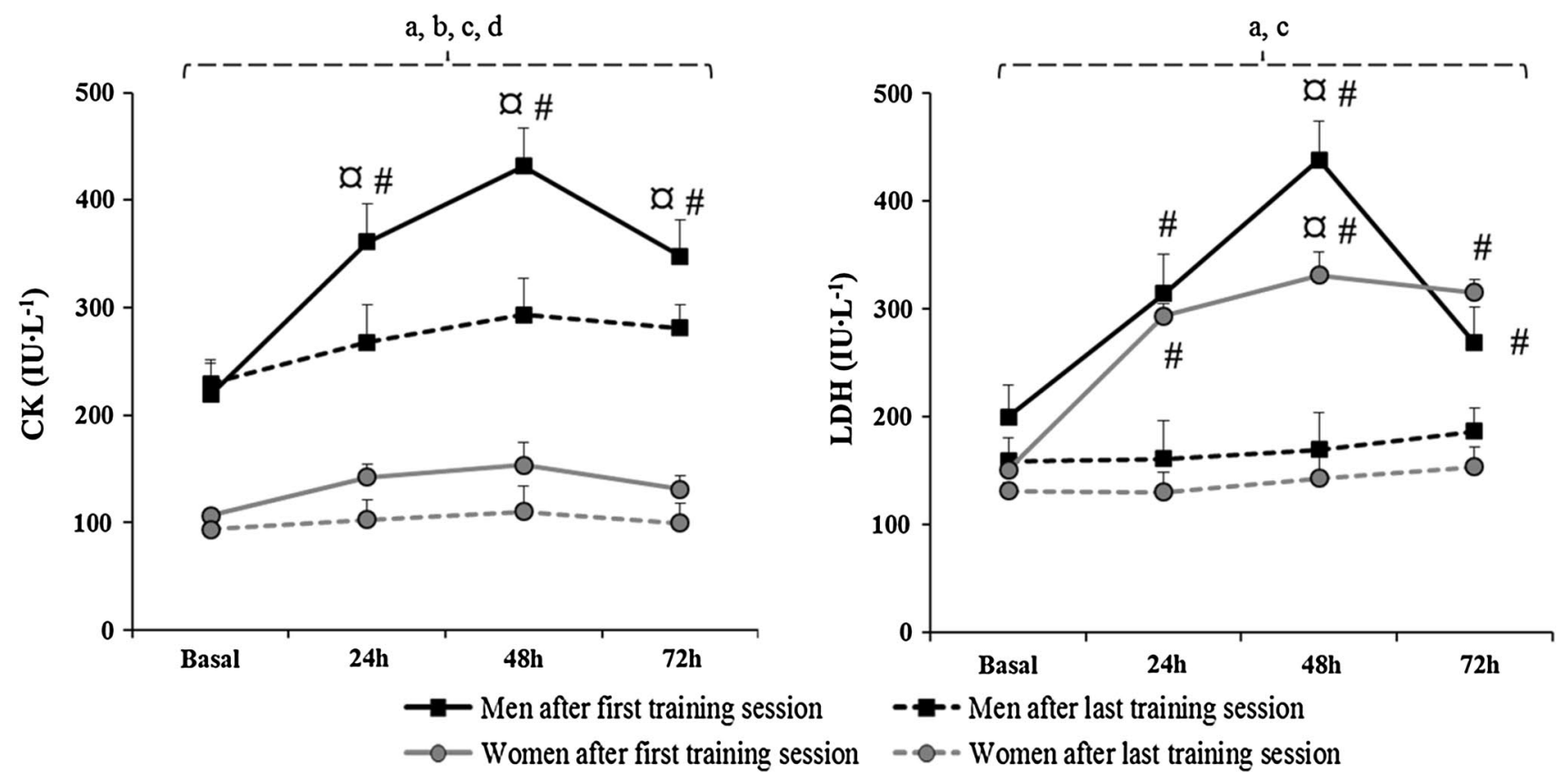

Fig. $5 \mathrm{CK}$ and LDH plasma concentrations before (basal) and 24, 48 and $72 \mathrm{~h}$ after the first and the last training sessions. Significant effects $(P<0.05): a$ training $\times$ time interaction, $b$ sex $\times$ time interac-

women, the CK concentration was 2.8 -fold higher, suggesting that other factors such as higher levels of estrogen in women (Tiidus 2000; Velders and Diel 2013) may account for the sex-based differences in CK. Regardless of explanation, the increase in markers of muscle damage in the current study was lower than in several other ECC-based exercises comprising similar number of repetitions per session. For example, acute bouts of isolated isokinetic ECC elbow flexions resulted in $\mathrm{CK}$ values over 2,000 $\mathrm{IU} \mathrm{L}^{-1}$ (Barroso et al. 2010; Chen et al. 2009), i.e., 4 times higher than the values reported in here. Differences in muscle damage responses across studies may perhaps be explained by the different contraction velocity regimen employed or study design factors (e.g., testing schedule). Although widely used, it should be acknowledged that serum levels of CK and LDH as sole markers of the muscle damage may not provide enough data to fully determine the muscle damage response after RE. Indeed, data obtained directly from analysis of muscle tissue (i.e., muscle biopsies) could have provided more detailed information about potential differences in muscle damage after ECC-overload RE across sexes.

Despite that peak power during the last exercise session was $\sim 50 \%$ greater than during the first, selected markers of muscle damage were not evident. Thus, even with the high forces employed during the current RE paradigm, the ECC overload did not provoke a chronic increase in markers of muscle damage in men or women. These results are tion, $c$ main effect of training, $d$ main effect of sex. Significant post hoc differences $(P<0.05)$ : ${ }^{\#}$ first vs last training session; $\propto$ vs basal value

consistent with the responses noted after conventional RE training with isolated ECC muscle actions (Neme Ide et al. 2013). Further, the finding is important because if the ECC load is not tolerated, markers of muscle damage may be present until completion of the training program, ultimately compromising muscle function and adaptations (Krentz and Farthing 2010).

ECC-overload RE training induced robust gains in maximal strength in both sexes, yet improvements were somewhat greater for men compared with women. In contrast, other RE paradigms have shown similar (Abe et al. 2000; Colliander and Tesch 1991), or greater relative strength gains in women (Hubal et al. 2005; O'Hagan et al. 1995). It is worth noting, however, that the greater capacity of women to increase strength after RE training was reported for the elbow flexor muscles in relative values (Hubal et al. 2005; O'Hagan et al. 1995). In this exercise, women are much weaker than men (Miller et al. 1993), which could explain the greater relative strength gains in women in those particular studies (Hubal et al. 2005; O'Hagan et al. 1995). Nevertheless, in the current investigation men increased $1 \mathrm{RM}$ to a greater extent than women. Given that relative increases in muscle mass did not show any sex difference, neural factors (e.g., increased neural drive, altered motor unit firing rate, enhanced motor unit synchronization) could perhaps account for the superior strength gains found in men. The magnitude of strength gains reported here is comparable to other studies employing longer 
(>10 weeks) training periods (Abe et al. 2000; Petrella et al. 2007). Thus, it appears that strength improvements are greater with ECC-overload RE compared with traditional weight training in both sexes (Abe et al. 2000; Petrella et al. 2007).

The training protocol did not induce gains in power at very low loads ( $40 \%$ of $1 \mathrm{RM}$ ) in any group, but it resulted in similar gains in power across groups at low-to-medium loads $(50,60,70 \%$ of $1 \mathrm{RM})$. Interestingly, power increments at $80 \%$ of $1 \mathrm{RM}$ were greater for men than women. Similarly, Colliander and Tesch (1991) reported greater power improvements for men than women after maximal CON-ECC isokinetic training. Conversely, when no maximal (or overloaded) ECC muscle actions were employed, power increased similarly in men and women (Delmonico et al. 2005). While these notions suggest that, for unknown reasons, sex-based differences in power adaptations may preferentially occur when the ECC component is emphasized, ECC-overload training appears as an effective tool to increase power in both men and women. The increase in vertical jump performance reported here and elsewhere (Rittweger et al. 2007; Romero-Rodriguez et al. 2011), also supports this contention. It is noteworthy, however, that women showed greater increase in jump height than men. Although this difference ( 8 vs $4 \%$ ) was not statistically significant, it contrasts with the power gains at high loads (i.e., $80 \%$ of $1 \mathrm{RM}$ ), where men outscored women. Thus, even when the training stimulus is similar across sexes, men and women may show subtle differences in power adaptations. Future studies addressing this issue are warranted.

Whether or not sex-based differences in muscle hypertrophy induced by RE exist is unclear. Thus, inconclusive (Abe et al. 2000; O'Hagan et al. 1995) reports suggest greater increases in muscle size in men compared with women (Hubal et al. 2005; Melnyk et al. 2009). While the efficacy of ECC-overload RE to induce muscle hypertrophy in men and women has been well documented (Lundberg et al. 2013; Norrbrand et al. 2008; Tesch et al. 2004), specific examination across sexes had not yet been performed. Results from the current study show comparable $(\sim 5 \%)$ increases in muscle mass in both men and women, concerting the rate of muscle hypertrophy typically reported after flywheel knee extension RE (Norrbrand et al. 2008; Tesch et al. 2004). Thus, although differences between single- and multi-joint exercises should not be excluded, we conclude that ECC-overload RE induces robust muscle hypertrophy in both men and women. In further support, previous training studies (Abe et al. 2000; O'Hagan et al. 1995) have also shown comparable hypertrophy responses across sexes, despite differences in hormonal and/or cellular responses after high-intensity exercise between men and women (Esbjornsson et al. 2012; Kraemer et al. 1998).
In summary, the present investigation assessed strength, power, muscle mass, and muscle damage responses after short-term ECC-overload RE training in men and women. Although men showed slightly greater gains in $1 \mathrm{RM}$ and power at $80 \%$ of $1 \mathrm{RM}$, marked improvements in dynamic strength and power were seen in both sexes. The increased muscle function was accompanied with comparable gains in muscle mass $(\sim 5 \%)$. While ECC-overload training induced muscle damage at the onset of training, the 6-week training period ameliorated this response in both sexes. Thus, untrained men and women adapt to short-term ECCoverload training by increasing strength, power and muscle mass with no concomitant adverse effects from muscle damage.

Acknowledgments This study was partly funded by Consejería de Educación, Junta de Castilla y León, Spain.

Conflict of interest The authors declare no conflicts of interest.

\section{References}

Abe T, DeHoyos DV, Pollock ML, Garzarella L (2000) Time course for strength and muscle thickness changes following upper and lower body resistance training in men and women. Eur J Appl Physiol 81(3):174-180. doi:10.1007/s004210050027

Alkner BA, Tesch PA (2004a) Efficacy of a gravity-independent resistance exercise device as a countermeasure to muscle atrophy during 29-day bed rest. Acta Physiol Scand 181(3):345-357. doi:10.1111/j.1365-201X.2004.01293.x

Alkner BA, Tesch PA (2004b) Knee extensor and plantar flexor muscle size and function following 90 days of bed rest with or without resistance exercise. Eur J Appl Physiol 93(3):294-305. doi:10.1007/s00421-004-1172-8

Allen DG (2001) Eccentric muscle damage: mechanisms of early reduction of force. Acta Physiol Scand 171(3):311-319. doi:10.1046/j.1365-201x.2001.00833.x

Andreoli A, Scalzo G, Masala S, Tarantino U, Guglielmi G (2009) Body composition assessment by dual-energy X-ray absorptiometry (DXA). Radiol Med 114(2):286-300. doi:10.1007/ s11547-009-0369-7

Askling C, Karlsson J, Thorstensson A (2003) Hamstring injury occurrence in elite soccer players after preseason strength training with eccentric overload. Scand J Med Sci Sports 13(4):244-250

Barroso R, Roschel H, Ugrinowitsch C, Araujo R, Nosaka K, Tricoli $\mathrm{V}$ (2010) Effect of eccentric contraction velocity on muscle damage in repeated bouts of elbow flexor exercise. Appl Physiol Nutr Metab 35(4):534-540. doi:10.1139/H10-042

Berg HE, Tesch A (1994) A gravity-independent ergometer to be used for resistance training in space. Aviat Space Environ Med 65(8):752-756

Billaut F, Smith K (2009) Sex alters impact of repeated bouts of sprint exercise on neuromuscular activity in trained athletes. Appl Physiol Nutr Metab 34(4):689-699. doi:10.1139/H09-058

Cannon JG, Meydani SN, Fielding RA, Fiatarone MA, Meydani M, Farhangmehr M, Orencole SF, Blumberg JB, Evans WJ (1991) Acute phase response in exercise. II. Associations between vitamin E, cytokines, and muscle proteolysis. Am J Physiol 260(6 Pt 2):1235-1240 
Chen TC, Chen HL, Lin MJ, Wu CJ, Nosaka K (2009) Muscle damage responses of the elbow flexors to four maximal eccentric exercise bouts performed every 4 weeks. Eur J Appl Physiol 106(2):267-275. doi:10.1007/s00421-009-1016-7

Colliander EB, Tesch PA (1990) Effects of eccentric and concentric muscle actions in resistance training. Acta Physiol Scand 140(1):31-39

Colliander EB, Tesch PA (1991) Responses to eccentric and concentric resistance training in females and males. Acta Physiol Scand 141(2):149-156

Colliander EB, Tesch PA (1992) Effects of detraining following short term resistance training on eccentric and concentric muscle strength. Acta Physiol Scand 144(1):23-29

Delmonico MJ, Kostek MC, Doldo NA, Hand BD, Bailey JA, RabonStith KM, Conway JM, Carignan CR, Lang J, Hurley BF (2005) Effects of moderate-velocity strength training on peak muscle power and movement velocity: do women respond differently than men? J Appl Physiol 99(5):1712-1718. doi:10.1152/japplp hysiol.01204.2004

Dudley GA, Tesch PA, Harris RT, Golden CL, Buchanan P (1991a) Influence of eccentric actions on the metabolic cost of resistance exercise. Aviat Space Environ Med 62(7):678-682

Dudley GA, Tesch PA, Miller BJ, Buchanan P (1991b) Importance of eccentric actions in performance adaptations to resistance training. Aviat Space Environ Med 62(6):543-550

Esbjornsson M, Rundqvist HC, Mascher H, Osterlund T, Rooyackers O, Blomstrand E, Jansson E (2012) Sprint exercise enhances skeletal muscle p70S6 k phosphorylation and more so in women than in men. Acta Physiol (Oxf) 205(3):411-422. doi:10.1111/j.1748-1716.2012.02404.x

Fernandez-Gonzalo R, De Paz JA, Rodriguez-Miguelez P, Cuevas MJ, Gonzalez-Gallego J (2012) Effects of eccentric exercise on toll-like receptor 4 signaling pathway in peripheral blood mononuclear cells. J Appl Physiol 112(12):2011-2018. doi:10.1152/ja pplphysiol.01499.2011

Garcia-Lopez D, Cuevas MJ, Almar M, Lima E, De Paz JA, Gonzalez-Gallego J (2007) Effects of eccentric exercise on NF-kappaB activation in blood mononuclear cells. Med Sci Sports Exerc 39(4):653-664. doi:10.1249/mss.0b013e31802f04f6

Golden CL, Dudley GA (1992) Strength after bouts of eccentric or concentric actions. Med Sci Sports Exerc 24(8):926-933

Hansen RD, Williamson DA, Finnegan TP, Lloyd BD, Grady JN, Diamond TH, Smith EUR, Stavrinos TM, Thompson MW, Gwinn TH (2007) Estimation of thigh muscle cross-sectional area by dual-energy X-ray absorptiometry in frail elderly patients. Am J Clin Nutr 86(4):952

Hather BM, Tesch PA, Buchanan P, Dudley GA (1991) Influence of eccentric actions on skeletal muscle adaptations to resistance training. Acta Physiol Scand 143(2):177-185

Hubal MJ, Gordish-Dressman H, Thompson PD, Price TB, Hoffman EP, Angelopoulos TJ, Gordon PM, Moyna NM, Pescatello LS, Visich PS, Zoeller RF, Seip RL, Clarkson PM (2005) Variability in muscle size and strength gain after unilateral resistance training. Med Sci Sports Exerc 37(6):964-972

Kraemer WJ, Staron RS, Hagerman FC, Hikida RS, Fry AC, Gordon SE, Nindl BC, Gothshalk LA, Volek JS, Marx JO, Newton RU, Hakkinen K (1998) The effects of short-term resistance training on endocrine function in men and women. Eur J Appl Physiol Occup Physiol 78(1):69-76

Krentz JR, Farthing JP (2010) Neural and morphological changes in response to a 20-day intense eccentric training protocol. Eur J Appl Physiol 110(2):333-340. doi:10.1007/s00421-010-1513-8

Lundberg TR, Fernandez-Gonzalo R, Gustafsson T, Tesch PA (2013) Aerobic exercise does not compromise muscle hypertrophy response to short-term resistance training. J Appl Physiol 114(1):81-89. doi:10.1152/japplphysiol.01013.2012
Mastaloudis A, Traber MG, Carstensen K, Widrick JJ (2006) Antioxidants did not prevent muscle damage in response to an ultramarathon run. Med Sci Sports Exerc 38(1):72-80. doi:00005768-200601000-00012

Melnyk JA, Rogers MA, Hurley BF (2009) Effects of strength training and detraining on regional muscle in young and older men and women. Eur J Appl Physiol 105(6):929-938. doi:10.1007/ s00421-008-0979-0

Milias GA, Nomikos T, Fragopoulou E, Athanasopoulos S, Antonopoulou S (2005) Effects of eccentric exercise-induced muscle injury on blood levels of platelet activating factor (PAF) and other inflammatory markers. Eur J Appl Physiol 95(5-6):504-513. doi:10.1007/s00421-005-0031-6

Miller AE, MacDougall JD, Tarnopolsky MA, Sale DG (1993) Gender differences in strength and muscle fiber characteristics. Eur J Appl Physiol Occup Physiol 66(3):254-262

Neme Ide B, Alessandro Soares Nunes L, Brenzikofer R, Macedo DV (2013) Time course of muscle damage and inflammatory responses to resistance training with eccentric overload in trained individuals. Mediat Inflamm 2013:204942. doi:10.1155/2013/204942

Norrbrand L, Fluckey JD, Pozzo M, Tesch PA (2008) Resistance training using eccentric overload induces early adaptations in skeletal muscle size. Eur J Appl Physiol 102(3):271-281. doi:10.1007/s00421-007-0583-8

Norrbrand L, Pozzo M, Tesch PA (2010) Flywheel resistance training calls for greater eccentric muscle activation than weight training. Eur J Appl Physiol 110(5):997-1005. doi:10.1007/ s00421-010-1575-7

Norrbrand L, Tous-Fajardo J, Vargas R, Tesch PA (2011) Quadriceps muscle use in the flywheel and barbell squat. Aviat Space Environ Med 82(1):13-19

Nosaka K, Newton M (2002) Concentric or eccentric training effect on eccentric exercise-induced muscle damage. Med Sci Sports Exerc 34(1):63-69

O'Hagan FT, Sale DG, MacDougall JD, Garner SH (1995) Response to resistance training in young women and men. Int J Sports Med 16(5):314-321. doi:10.1055/s-2007-973012

Petrella JK, Kim JS, Tuggle SC, Bamman MM (2007) Contributions of force and velocity to improved power with progressive resistance training in young and older adults. Eur J Appl Physiol 99(4):343-351. doi:10.1007/s00421-006-0353-z

Ploutz-Snyder LL, Giamis EL, Formikell M, Rosenbaum AE (2001) Resistance training reduces susceptibility to eccentric exerciseinduced muscle dysfunction in older women. J Gerontol A Biol Sci Med Sci 56(9):384-390

Rittweger J, Felsenberg D, Maganaris C, Ferretti JL (2007) Vertical jump performance after 90 days bed rest with and without flywheel resistive exercise, including a 180 days follow-up. Eur J Appl Physiol 100(4):427-436. doi:10.1007/ s00421-007-0443-6

Romero-Rodriguez D, Gual G, Tesch PA (2011) Efficacy of an inertial resistance training paradigm in the treatment of patellar tendinopathy in athletes: a case-series study. Phys Ther Sport 12(1):43-48. doi:10.1016/j.ptsp.2010.10.003

Schroeder ET, Hawkins SA, Jaque SV (2004) Musculoskeletal adaptations to 16 weeks of eccentric progressive resistance training in young women. J Strength Cond Res 18(2):227-235. doi: $10.1519 / \mathrm{r}-13443.1$

Shephard RJ (2000) Exercise and training in women, part I: influence of gender on exercise and training responses. Can J Appl Physiol 25(1):19-34

Staron RS, Karapondo DL, Kraemer WJ, Fry AC, Gordon SE, Falkel JE, Hagerman FC, Hikida RS (1994) Skeletal muscle adaptations during early phase of heavy-resistance training in men and women. J Appl Physiol 76(3):1247-1255 
Stupka N, Lowther S, Chorneyko K, Bourgeois JM, Hogben C, Tarnopolsky MA (2000) Gender differences in muscle inflammation after eccentric exercise. J Appl Physiol 89(6):2325-2332

Stupka N, Tarnopolsky MA, Yardley NJ, Phillips SM (2001) Cellular adaptation to repeated eccentric exercise-induced muscle damage. J Appl Physiol 91(4):1669-1678

Tesch PA, Ekberg A, Lindquist DM, Trieschmann JT (2004) Muscle hypertrophy following 5-week resistance training using a non-gravity-dependent exercise system. Acta Physiol Scand 180(1):89-98

Thompson HS, Scordilis SP (1994) Ubiquitin changes in human biceps muscle following exercise-induced damage. Biochem Biophys Res Commun 204(3):1193-1198. doi:10.1006/b brc. 1994.2589
Tiidus PM (2000) Estrogen and gender effects on muscle damage, inflammation, and oxidative stress. Can J Appl Physiol 25(4):274-287

Trappe TA, Burd NA, Louis ES, Lee GA, Trappe SW (2007) Influence of concurrent exercise or nutrition countermeasures on thigh and calf muscle size and function during 60 days of bed rest in women. Acta Physiol (Oxf) 191(2):147-159. doi:10.1111/j.1748-1716.2007.01728.x

Velders M, Diel P (2013) How sex hormones promote skeletal muscle regeneration. Sports Med 43(11):1089-1100. doi:10.1007/ s40279-013-0081-6 